\title{
Los procesos sustractivos de empleo (II)
}

\author{
Por Luis Puchol*
}

\subsection{PROCESOS SUSTRACTIVOS MENOS FRECUENTES}

La aplicación de soluciones imaginativas a los procesos sustractivos de empleo, además de enfrentarse a la triple barrera a que me refería anteriormente (rigidez de la normativa laboral, resistencia de los empresarios al cambio, desconfianza de los trabajadores) exige, por parte de todas las partes afectadas, una ruptura del esquema mental que tradicionalmente se ha tenido de lo que es un empleo, y su sustitución por un concepto más actual y más dinámico que ya se va abriendo paso entre legisladores, empresarios y asalariados.

El esquema tradicional de empleo, según PUEL (32), reúne las siguientes características:

- El empleo típico es un empleo asalariado.

- En él, el vínculo salarial es firme.

- El empleo típico es estable y debe permitir una carrera.

- El empleo típico lo es a tiempo completo.

- El empleo típico procura lo esencial de los ingresos familiares.

- El empleo típico depende de un solo patrono.

- El empleo típico se ejerce en un lugar específico.

- En el empleo típico, el empleado tiene toda y sólo la responsabilidad de su puesto de trabajo.

Cuando se afirma que en la actualidad no es posible, ni siquiera soñar, en la situación de pleno empleo, es a este preciso modelo al que nos estamos refiriendo. Pero sí existen posibilidades de empleo para todos, o al menos para muchos más trabajadores de los que actualmente disponen de un puesto de trabajo, a condición de que reformulemos la definición de qué cosa es un empleo.

* Profesor de Dirección de Personal en la Facultad de Ciencias Económicas y Empresariales de la Universidad Comillas (ICADE).

(32) PUEL. H. "Il y a emploi et emploi". Travail et Emploi. Avril 1980, n." 4. 
Afirma GOGUELIN (33) que "el empleo es el sistema de las relacio" nes entre el hombre que cambia y el trabajo que se modifica en un contexto económico y psicosocial (los otros hombres de la empresa, la empresa, la sociedad, la cultural que está en perpetuo devenir".

Por su parte SAGARDOY comenta la necesidad de adaptar los instrumentos jurídicos al cambio de filosofía en cuanto at empleo, de acuerdo con las previsiones de la O.l.T. Estos cambios se resumen en el desplazamiento del énfasis recayente sobre la continuidad en el empleo, a la seguridad en el empleo. Dicho de otro modo, se trata de poner en práctica una política legislativa "que no pretenda, como fin último, el carácter indefinido de la relación laboral, sino más bien la búsqueda de mecanismos que realicen una estabilidad profesional, por encima de la estabilidad en el puesto de trabajo, e incluso en la empresa" (34).

Si es cierto (y seguro que lo es) que la única constante en el mundo actual es el cambio, es lógico pensar que los remedios de antaño no sirvan para resolver los complejos problemas de hogaño. Los individuos. las organizaciones, las sociedades, las civilizaciones, que son capaces de prever el cambio y de adaptarse a él, sobreviven y prosperan, pero la historia nos refiere el triste destino de aquellas civilizaciones, aquellas sociedades y aquellos pueblos que, faltos de la capacidad de reacción, sucumbieron y fueron desplazados por otros pueblos y otras culturas más adaptadas a las condiciones mutantes.

Las páginas siguientes no son un recetario, ni tampoco pretenden constituirse en relación exhaustiva de las posibilidades existentes para solucionar el problema del paro. Pretenden, eso sí, dar una visión forzosamente sintética y superficial de lo que hemos denominado procesos sustractivos de empleo. Si la aplicación a nivel micro de estas soluciones coadyuva a paliar a nivel macro el problema del desempleo, todos nos felicitaremos por ello, pero quede bien entendido que le empresa, cada empresa en particular, ni siquiera el conjunto de ellas, no puede, ni debe, enfrentarse sola con la resolución de un problema de tales características, que es responsabilidad de la sociedad entera. La empresa, cada empresa, debe procurar su rentabilidad, o al menos su viabilidad. Esa es la manera más segura de contribuir al bienestar social. Como afirma el conocido proverbio, "si cada cual barre la puerta de su casa, la ciudad estará limpia".

Las soluciones esbozadas en la actualidad para la aplicación de los procesos sustractivos "menos frecuentes", se pueden clasificar en tres grandes grupos:

- Soluciones tendentes a la "descontratación" del personal, y a su reconversión en autónomos, suministradores, subcontratistas, o a su recolocación en otras empresas.

(33) GOGUELIN, P. "L'analyse d'un universitaire", Personnel. Mars-avril 1981, n. 231. (Número monográfico dedicado a los problemas de empleo).

(34) SAGARDOY. A. "Medidas legales en favor del empleo". Papeles de Economía Española, n. $\dagger$ (Crisis Ecanómica) 1980, p. 157. 

trabajo.

- Soluciones basadas en el acondicionamiento del tiempo de

- Soluciones basadas en la reconversión interna.

\subsubsection{SOLUCIONES TENDENTES A LA "DESCONTRATACION" DEL PERSONAL Y A SU RECONVERSION EN AUTONOMOS, ETC.}

Con frecuencia hemos oído a trabajadores en paro referir su odisea personal. Comenzaron por ser despedidos por la aplicación de un expediente de regulación de empleo, o de reconversión. Percibieron una indemnización más o menos crecida en función de su antigüedad en la empresa y de la habilidad con que la representación sindical condujera las negociaciones, y pasaron a percibir el subsidio de desempleo. Al principio todo marchó bastante bien. La indemnización, incluso produjo ciertos efectos euforizantes (algunos gastaron parte de ella en cambiar de coche, o en acondicionar la vivienda familiar) y se dedicaron a buscar un nuevo empleo con criterios selectivos: un puesto, a ser posible, cerca del domicilio, con una remuneración no inferior a la del puesto anterior, por supuesto dentro de su propia especialidad u oficio. Conforme iba transcurriendo el tiempo, las exigencias iniciales fueron suavizándose gradualmente, para - al cabo de algunos meses - agotada ya la inyección económica que supuso la indemnización, $y$ ante la perspectiva de la finalización del periodo de percepción del subsidio del desempleo, estar dispuestos a aceptar cualquier trabajo, sin conseguir su propósito.

Frente a esta triste realidad, una constatación paradójica: en el paro. buscando infructuosamente un puesto de trabajo asalariado hay cantidad de pintores, fontaneros, carpinteros y demás profesionales de oficio, y sin embargo cualquiera puede atestiguar la dificultad de encontrar a uno de estos profesionales para pintar un piso, acuchillar el parquet o hacer una estantería a la medida.

Si los trabajadores despedidos, a nivel individual o colectivo, hubieran empleado sus indemnizaciones de despido en establecerse como trabajadores autónomos, o en constituir una sociedad (cooperativa o no) es posible que hubieran podido mantener un status similar al anterior, o incluso superior, toda vez que - como autónomos - no tendrían ninguna limitación de horas de trabajo.

Si en otra época de industrialización creciente, las formas de autoempleo fueron reduciéndose, a la par que marginándose, es posible que haya sonado la hora de invertir el signo. Pasar de la categoría de asalariado a la de autónomo, a costa de perder - eso sí- la seguridad que da un empleo (que, a la hora de la verdad, vemos que era más ilusoria que real).

Es posible que las empresas se resistan a contratar a un conductor de motocarro para realizar el reparto de pequeña paquetería, pero necesitan a alguien que realice este servicio, y están dispuestas a establecer un arreglo con un autónomo, en exclusiva o compartido con otras empresas. 
El paso siguiente, por parte de las empresas que se encuentran con una plantilla sobredimensionada es la de suprimir aquellas secciones que resultan deficitarias. A veces, los déficits obedecen más a causas de conflictividad o de problemas interpersonales, que a motivos de falta de trabajo. El camino habitual de expediente de regulación de empleo o el despido, ya lo conocemos. Se trata ahora de procurar la "descontratación" de los trabajadores excedentes con el menor gasto posible y de la manera menos traumática. Dos son los procedimientos utilizables:

- Conversión del personal contratado en suministradores o subcontratistas.

- El "out-placement".

a) Conversión del personal en suministradores...

Básicamente consiste en vender el departamento con su utillaje y servicios a los trabajadores, y luego contratar los servicios de éstos en calidad de subcontratistas independientes, bien sea en exclusiva, bien sea dejándole en libertad de elegir sus propios clientes, con la promesa de un tratamiento preferencial a los antiguos patronos. Lo habitual es que exista un período de exclusiva, que se suele cifrar en dos o tres años, para dar a todos la oportunidad de acostumbrarse a la nueva situación.

De este modo, la empresa paga por contrato comercial, en lugar de hacerlo por salarios. El resultado suele ser aceptablemente satisfactorio para una y otra parte. La empresa reduce gastos generales, aumenta su productividad y se libera de problemas laborales, siempre enojosos. Los empleados, que ahora trabajan para sí, en lugar de hacerlo para un empresario, miran más por el rendimiento y no regatean esfuerzos, con lo que normalmente suelen incrementar sus ingresos. Incluso se produce un curioso, pero nada sorprendente, viraje de valores. El trabajador "rácano", que antes era tolerado, cuando no admirado, es ahora censurado y conminado a corregir su postura.

La dificultad para llevar a cabo esta "descontratación" radica principalmente en la falta de flexibilidad legal y en la desconfianza de los trabajadores, quienes temen - con razón-dar un salto en el vacío. Por ello, el éxito de este tipo de operaciones depende en gran medida del acierto en "vender" la idea a los candidatos a la descontratación, a los que se debe garantizar la posibilidad de volver a la situación de asalariados si, al cabo de un tiempo de prueba, no se encontraran satisfechos con el cambio.

Existen experiencias de "descontratación" en campos muy diversos, que van desde el tratamiento informático a la industria de la confección pasando por la fotocomposición, el mantenimiento de maquinaria, la subcontratación del servicio de limpieza, o de vigilancia, el servicio del comedor o de la cafetería de empresa, etc. (35).

(35) Para una ampliación sobre el tema, con ejemplos concretos de "descontratación" en empresas españolas, vid. CLUTTERBUCK, D. "Como convertir rentablemente al personal en suministradores". International Management julio 1983, pp. 33-35. 
Una posibilidad que puede - o no- darse, es la de constituir a los trabajadores en cooperativa de trabajo asociado, pudiendo obtener los socios cooperadores unos créditos a cargo del Fondo Nacional de Protección al Trabajo, que resultan bastante atractivos, tanto por el bajo tipo de interés aplicado, como por el largo plazo de amortización (36).

El cooperativismo, que ha llegado a ser saludado como la síntesis feliz que venía a superar el enfrentamiento dialéctico capital-trabajo, en realidad no siempre ha sido entendido, ni por los capitalistas, ni por los trabajadores.

La cooperación obrera satisface una de las aspiraciones de los trabajadores: la autogestión. Proporciona una difusión del poder entre todos los socios y permite el libre ejercicio de los derechos sobre el producto del propio trabajo. Esta formulación resulta sospechosa para la mentalidad capitalista, que tolera la existencia de cooperativas, pero que las somete a una normativa legal poco incentivadora.

Por otra parte, la cooperación es vista con preocupación por los sindicatos" de clase". Temen éstos a la "desmovilización" de los trabajadores que la transformación de obreros asalariados en cooperativistas supone: son menos militantes dispuestos para la lucha de clases. Por otra parte, una vez constituidos en empresa, temen los sindicatos, que los cooperativistas se dediquen más a la resolución de sus problemas como grupo que a conseguir el cambio social para todos. La existencia de los principios de autogestión interna, no es para los sindicatos garantía de que, antes o después, no vayan a caer en el juego capitalista de la competencia y de la economía de mercado.

Por estas razones, la descontratación de trabajadores para su reconversión en cooperativistas suele contar con la resistencia pasiva de una legislación poco facilitadora, y la activa de unos comités de empresa que posiblemente tratarán de disuadir a los futuros cooperativistas por todos los medios a su alcance.

\section{b) El out-placement}

El out-placement es una práctica que se originó en los Estados Unidos en 1969 (37), y que consiste fundamentalmente en la contratación por parte de la empresa de los servicios de una agencia especializada, para que asesore en la readaptación y en la recolocación a aquellos directivos que van a quedarse sin trabajo.

(36) Vid. MOLERO, C. Op. cit. p. 105.

Para un tratamiento específico de la problemática del cooperativismo en España, vid. ARCO, J. del, "El Cooperativismo Español en la hora presente". Revista de Fomento Social, n. 149 , enero-marzo 1983, pp. 57-75.

Lo mismo, a nivel europeo, se encuentra en "Les sociétés ouvrières de production". Les cahiers de l'actualité religieuse et sociale. n. ${ }^{\circ} 87.1974$.

(37) Por George Hubber, de la consultora Think de Nueva York. 
La contratación de estos servicios a cargo de la empresa suele formar parte del "paquete de indemnizaciones" que ésta ofrece a un directivo por la pérdida de su empleo. En muchos casos, la indemnización va más allá de lo exigible legalmente, y tiende a tratar de compensar la dedicación de personas que fueron valiosas en un pasado, y que se desearía conservar en la empresa, si las circunstancias lo permitieran.

El out-placement es normalmente bien acogido por todos: a la empresa le supone una reducción de sus gastos de indemnización, a la vez que contribuye a crear una imagen de "empresa social" y ayuda a superar la "mala conciencia" que todo despido de un empleado fiel produce. El directivo que se marcha suele preferir un empleo de acuerdo con sus aptitudes y preparación que una indemnización, por elevada que ésta sea. Muchos directivos se niegan al simulacro de un despido improcedente, y detestan la humillación que para ellos supondría inscribirse en una oficina de empleo, y prefieren esta solución a cualquier otra. Los sindicatos, al tratarse de personal directivo, se inhiben y no suelen plantear problemas.

El out-placement clásico se aplica exclusivamente a la recolocación de directivos, pero cabría pensar en extender estos procesos a empleados y trabajadores de otros niveles. Desgraciadamente, en España no existen hasta el momento agencias que practiquen el out-placement, e incluso su viabilidad legal es problamática, debido al obstáculo que supone el artículo 40.2 de la Ley Básica de Empleo, que prohíbe la existencia de agencias privadas de colocación de cualquier clase, dando el monopolio de estas actividades al no siempre eficaz Instituto Nacional de Empleo, a través de sus Oficinas de Colocación (38).

La versión japonesa del out-placement, se fundamenta en el principio del shushin-koyo, o empleo vitalicio, combinado con el oficialmente desaparecido principio del Zaibatsu (holdings, o grupos de empresas vinculadas entre $\mathrm{s} f$ ). Cuando un directivo medio se jubila (a la edad de 55 años) recibe una indemnización y pasa a una empresa subsidiaria, en un trabajo a tiempo parcial durante diez ańos. Si en lugar de trasladar a un empleado por llegar a la edad de jubilación, se hace por cierre de una unidad de producción, tenemos el mismo principio que inspira el outplacement norteamericano, con la salvedad de que, en el Japón, se realiza por medios propios, sin recurrir a agencias externas, y por traslado a una sucursal del propio grupo (39).

(38) Este artículo, que reproduce el art: 16.2 del E. T., se fundamenta en los convenios $34 y$ 96 (revisado) de la OlT, ratificados ambos por Espafáa. En el Convenio 96 existe una doble alternativa: la supresión de las agencias de colocación retribuidas (alternativa suscrita por España). o reglamentación de las mismas. Si se deseara suscribir la segunda alternativa en lugar de la primera, había que esperar al año 1991, ya que el instrumento de ratificación fue depositado en 1971, y no se denunció al cumpir el período de diez años, quedando automáticamente obligado por un nuevo período de diez años.

(39) Para un conocimiento de las prácticas de empleo japonesas, resulta imprescindible la lectura del libro de OUCHI, W. "Teorla $Z$ ". Orbis. Barcelona, 1984.

Vid. igualmente INOHARA, H. "Mitos y realidades de la Dirección de Personal en el Japón". /CADE, n."2-84, pp. 59-78. 


\subsubsection{SOLUCIONES BASADAS EN EL ACONDICIONAMIENTO DEL TIEMPO DE TRABAJO}

El empleo típico ocupa la mayor parte de la vida consciente del individuo, y representa para la persona la posibilidad mayor de expresión de su personalidad, de participación en la vida social, de autorrealización, en suma.

La noción de tiempo de trabajo, para DETRAZ (40), se inscribe en un universo tridimensional: cronométrico, cronológico y psicológico.

La dimensión cronométrica mide de una manera cuantitativa el tiempo de trabajo de un asalariado (diario, semanal, mensual...), así como la duración de un turno, de una secuencia de producción, de una tarea precisa que ha de ser realizada. Se relaciona con la noción de intensidad de un trabajo.

La dimensión cronológica distribuye el tiempo en el espacio (duración de la vida activa, edad de la jubilación...) y reparte la vida del hombre en tres etapas: instrucción, actividad profesional, jubilación.

La dimensión psicológica, que es puramente subjetiva, difiere bastante de las dos precedentes. A menudo se olvida esta dimensión en los estudios de tiempo de trabajo, a pesar de que - dice DETRAZ - marca la separación entre aquellos que "emplean su tiempo en el trabajo" y los que "pierden su tiempo en el trabajo". Ciertamente, el tiempo que un ingeniero dedica a resolver un problema técnico, no es el mismo que el de un trabajador de la cadena que realiza una tarea parcelaria y repetitiva, $y$ para ser completos - añade DETRAZ - habría que tener en cuenta un parámetro más, de la mayor importancia: el de la edad.

Durante el siglo $X X$, la idea de lo que es un trabajo a tiempo completo ha ido evolucionando desde las 72 horas semanales, a las 40 actuales, 0 a las 35 demandadas por las centrales sindicales. Se puede afirmar que la reducción de tiempo de trabajo se ha desarrollado en cuatro direcciones:

- Reducción de días trabajados por semana (de una semana de seis días, a una semana de cinco días).

- Reducción de horas trabajadas por día (de 12 o 14 horas diarias a 8 o 7 horas/día).

- Reducción del período de vida activa (de jubilación a edades muy avanzadas a edades cada vez más tempranas y -paralelamenteretraso de la edad de incorporación al mundo del trabajo).

- Adaptación del tiempo de trabajo a las necesidades de la vida personal y social, lo que conduce al desmantelamiento de la normativa rigida $y$ uniforme en materia de tiempos de trabajo.

En Estados Unidos se define el trabajo típico por la fórmula $5 / 40$, esto es, cinco días de trabajo a la semana, con ocho horas de trabajo diarias. Esta semana típica, está saltando en pedazos en EE. UU., como va a suce-

(40) DETRAZ, A., "L'evolution du temps de travail et ses consequences sociales et syndican les". Persomel. Juillet-août 1982, n.2 243, pp. 18-24. 
der aquí, aunque los motivos sean bastante diferentes. Las razones que explican el acondicionamiento del tiempo de trabajo por parte de las empresas norteamericanas, se sintetizan principalmente en las siguientes:

- Agilizar las horas punta de entrada y de salida de los trabajadores (problemas de tráfico y de transporte público).

- Permitir a los trabajadores utilizar los senvicios recreativos y culturales de la comunidad, de una manera equilibrada, evitando los días de aglomeración y los días "vacíos".

- Compatibilizar el trabajo de fuera de casa, con el trabajo doméstico.

- Permitir a los trabajadores la prosecución de estudios, desarrollo de un deporte o "hobby".

- Etcétera.

NEWSTROM y PIERCE (41) resumen los cambios realizados en el tiempo de trabajo en los Estados Unidos, por medio del gráfico siguiente:

TIPOLOGIA DE LAS ALTERNATIVAS EN LOS HORARIOS DE TRABAJO

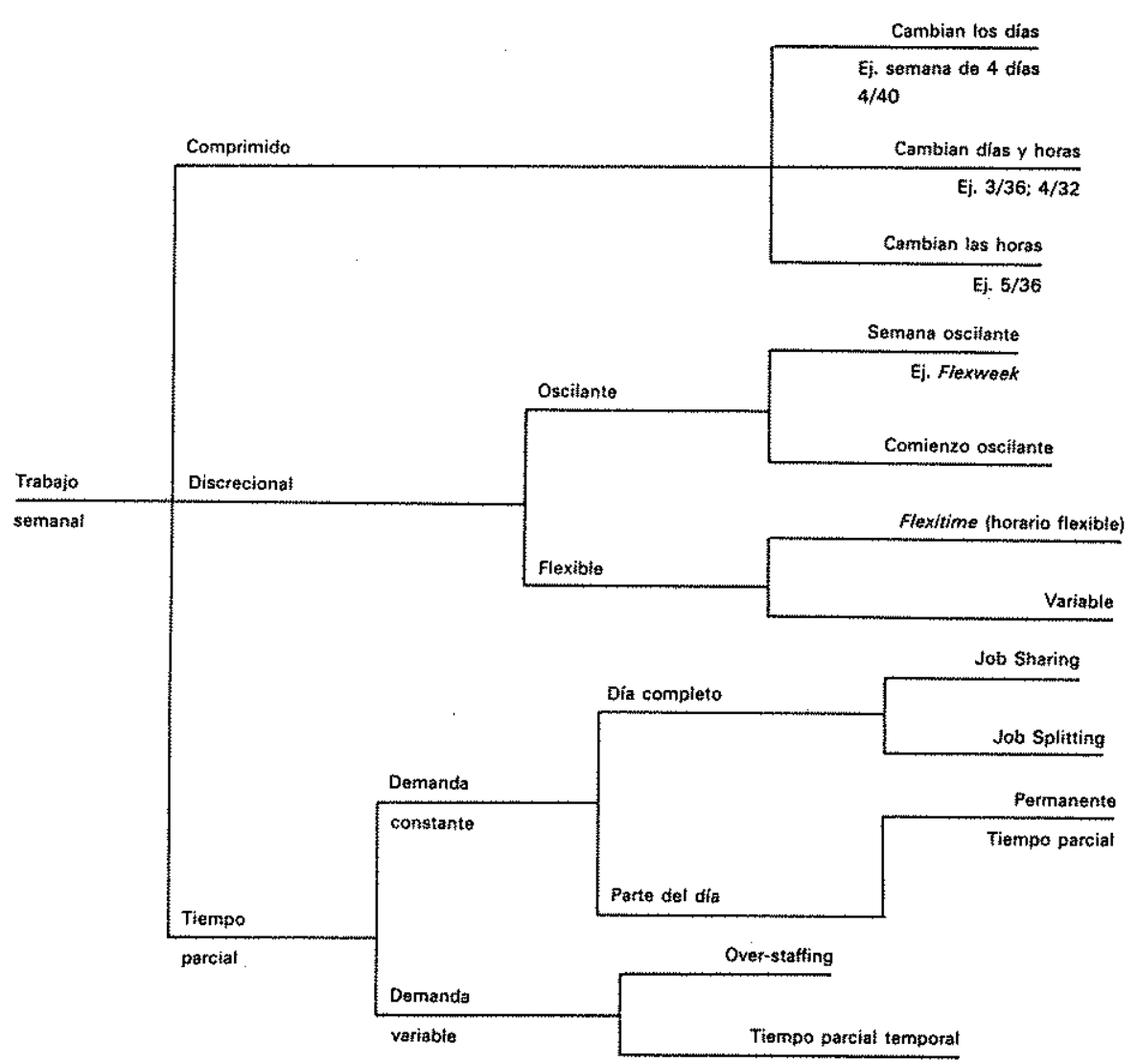

(41) NEWSTROM, J. W. Y PIERCE, J. L., "Alternative work schedules: the state of the art". The Personnell Administrator. October 1979 , pp. 19-23. 
Del extenso cuadro anterior, los únicos acondicionamientos horarios que pueden usarse con fines sustractivos son los de trabajo a tiempo parcial, de los que vamos a ocuparnos seguidamente (42).

El trabajo a tiempo parcial (TTP) parece ser la solución, no ideal, pero sí la menos mala, para satisfacer simultáneamente los objetivos económicos de la empresa y los objetivos sociales (proporcionar trabajo a quienes carecen de él, o responder a la demanda de aquellos que necesitan o desean compatibilizar el trabajo con diversas situaciones familiares y personales).

Es, además, la única solución para acomodar horarios irregulares en empresas cuyo proceso no se ajusta al "horario de oficinas". THIERRY (43) pone los siguientes ejemplos dé empresas que tienen que recurrir forzosamente al TTP:

- Industrias farmacéuticas con un horario de doce horas diarias, en lugar de ocho.

- Bancos que desean mantener las oficinas abiertas por las tardes, o abrir los sábados (44).

- Empresas lecheras que precisan funcionar 96 horas/semana, con un horario colectivo de $35 \mathrm{~h} / \mathrm{s}$.

Tradicionalmente, las fuerzas sindicales se han opuesto a cualquier forma de TTP, por cuanto estimaban que era una arma peligrosa que permitiría rebajar los salarios. La postura actual de los sindicatos refleja hoy en día el slogan ampliamente difundido por toda Europa: "Trabajar menos, para que trabajen todos". La pretensión de las fuerzas sindicales consiste en la reducción de jornada de 40 a 35 horas/semana, pero manteniendo la remuneración, postura analógicamente concordante - dicen-con la recomendación del convenio n. 047 de la 0.l.T., cuando se pretendía implantar la semana de las 40 horas, implantación que debería hacerse "en forma tal que no implique una disminución del nivel de vida de los trabajadores".

Por su parte, los empresarios están dispuestos a considerar las reducciones de jornada laboral, siempre que se parta del principio de una disminución paralela y proporcional de la retribución y de las cargas sociales, porque, arguyen, aunque los costes directos permanezcan constantes, los costes indirectos (reclutamiento y selección, adiestramiento y formación, administración de personal, inversiones, etc.) aumentan. De tal modo que lo justo sería que los sacrificios se repartieran

(42) Para un mejor conocimiento de los horarios comprimidos y discrecionales, de los que no vamos a tratar aquilí, vid.:

GLUECK. W. E., "Changing hours of work: a review and analysis of the research." The Personnel Administrator. March 1979, pp. 44-67.

MCCARTHY. M. "Trends in the development of alternative work patterns". The Personnel Administrator. October 1979, pp. 25-31.

BERNARD, K. E., "Flexitime's potential for management". The Personnel Administrator. October 1979, pp. 51-54.

"Vers une plus grande flexibilité du temps du travall". Intersocial. Mars 1982, n. 79.

(43) THIERRY, D., "Travall à temps partiel (ou a temps choisi)". Personnel. Sept, 1983, n." 253, pp. $11+14$.

(44) En Francia la banca sólo trabaja de lunes a viernes. 
entre la empresa, que se haría cargo del incremento de los costes indirectos, y de los trabajadores, que al reducir su jornada laboral renunciarían a una parte proporcional de su salario (45).

En todo caso, el desacuerdo entre las fuerzas sociales no es en absoluto radical, lo que, si se prosigue el camino iniciado de remover los obstáculos legales que dificultan estos tipos de contratación, hace suponer un futuro, ya cercano, en que este tipo de contratos gozará de amplio predicamento.

Pero para ello será necesario, una vez más, romper una serie de prejuicios acerca del TTP y de las personas que lo practican. En efecto, en la opinión colectiva, el TTP tiende a asimilarse a:

- Trabajo en puestos de escasa cualificación.

- Trabajo para mujeres.

- Trabajo para estudiantes.

- Bajas remuneraciones.

- Trabajadores que viven su situación laboral como un simple medio de subsistencia, y que no se implican demasiado.

En los momentos actuales, la idea de TTP es "vendible" a determinados colectivos de trabajadores:

- Algunas profesiones facilitan, e incluso imponen, actividades a tiempo parcial, y sus titulares, a menudo, adicionan varios, para reconstituir un trabajo a tiempo total.

La multiactividad, que no es lo mismo que el pluriempleo, es frecuente, desde hace tiempo, en la agricultura. En la Comunidad Valenciana, por ejemplo, donde la estructura de la propiedad agraria está tan alejada del minifundismo gallego como del latifundismo andaluz, no es infrecuente el caso de pequeñas propiedades agrícolas que, se han liberado de los trabajos más penosos, quedándoles tiempo libre para dedicarlo a otra actividad remunerada, no agrícola, La imagen de un automóvil remolcando una "mula mecánica" no es rara en los aparcamientos de las empresas fabriles rurales valencianas. Cuando acaben su jornada de trabajo en la fábrica, se acercarán a cultivar el "pedazo". Esta multiactividad condiciona también las relaciones laborales. El hecho de tratarse de pequeños propietarios, hace que sus posturas no sean tan radicales como las de aquellos que tienen que depender de su sueldo exclusivamente. Ahora bien, si llegan a declararse en huelga, su capacidad de resistencia es enorme, precisamente por la misma razón.

Otra singularidad en la conducta laboral de estos trabajadores es su escasa proletarización, y consiguiente baja afiliación y actividad sindical, así como un absentismo laboral pronunciado en las épocas que coinciden con determinadas tareas agrícolas.

Al margen de las agrarias, otras profesiones incluso liberales, tienden de manera natural a la multiactividad: los profesores, los psicólogos,

(45) Además, ésta es la fómula que recoge el art. ${ }^{\circ} 2.2$. del R. D. 1991/1984, de 31 de octubre, que regula este tipo de trabajo. 
graduados sociales, gestores, etc. suelen buscar soluciones de este tipo.

- Muchas mujeres casadas estarían dispuestas a compartir su puesto de trabajo con otra persona, para poder atender mejor los trabajos del hogar, la crianza de los hijos y la vida de familia.

- Determinadas personas, a partir de un cierto nivel de cualificación y remuneración, estarían dispuestas a "comprar tiempo libre" para proseguir estudios, desarrollar actividades no lucrativas o, simplemente, llevar una vida personal más agradable (46).

- Muchos matrimonios jóvenes estarian dispuestos a compartir un único puesto de trabajo entre los dos cónyuges, especialmente en los momentos presentes en que el valor "trabajo" cede ante otros valores en la mentalidad de las nuevas generaciones.

La idea tradicional es que el salario del cabeza de familia (varón) debía asegurar el mantenimiento de la unidad familiar. El reparto de roles en la pareja estaba perfectamente determinado: el marido debía traer un salario a casa, y la mujer era la encargada de administrarlo. Incluso en los casos en que la mujer trabajaba, se entendía que estos ingresos eran una "ayuda" para el salario principal. Tampoco era infrecuente que las ganancias de la mujer se ahorrasen y se destinaran a compras excepcionales (la renovación del mobiliario, la "entrada" para una vivienda, un viaje o unas vacaciones).

En la actualidad, una compleja amalgama de concausas tienden a borrar la distinción entre el salario "principal" del marido y el salario "secundario" de la mujer. Entre estas concausas cabe citar:

- Los cambios de rol en la pareja. Hoy no es raro, ni vergonzoso, el que el marido ayude a su esposa en los trabajos caseros, y no sólo en los que - por su dureza o necesidad de habilidad mecánica - se consideraron en un pasado como trabajos masculinos, sino también en los tradicionalmente femeninos (limpieza, compra, cocina, culdado de los niños pequeños...).

- El acceso de la mujer a niveles de educación equivalentes a los alcanzados por los varones, hace que los ingresos de ésta no sean una cantidad a despreciar, como propia de un trabajo no especializado:

- En algunos casos, el salario de la mujer se convierte en el principal, cuando no en el único. Por ejemplo, en los matrimonios formados por médico y enfermera - una combinación bastante habitual- con frecuencia la mujer "mantiene" al marido mientras éste prepara unas oposim ciones, realiza el doctorado o cursa la especialidad. Lo mismo cabe decir de los matrimonios de profesionales de otras ramas.

(46) El $88.3 \%$ de trabajadores alemanes, empleados a tiempo completo, estatian interesados en disminuir su tiempo de trabajo, a costa de experimentar una reducción paralela en sus ingresos, según mostró una encuesta realizada en 1979 por el IAB (Instituto para el estudio del mercado de trabajol de Nüremberg, sobre una muestra de 1.546 asalariados, empleados en las 200 profesiones y oficios más corrientes. 
La tendencia en los matrimonios jóvenes, parece que se va a decantar por la multiplicación de fuentes de percepción de rentas salariales, por medio del reparto de trabajos atípicos entre marido y esposa. ciones:

Dentro del TTP, podemos distinguir dos grandes grupos de solu-

- Aquellas en las que el trabajador realiza una jornada laboral de duración inferior en un tercio, al menos, de la jornada considerada normal y que -en consecuencia - percibe una retribución proporcional al tiempo trabajado.

- Aquellas otras fórmulas fundamentadas en el trabajo compartido, al que, para simplificar, denominaremos TC.

En el trabajo a tiempo compartido (TC), podemos encontrar tres situaciones ligeramente diferentes entre sí y que, por esta misma razón, son frecuentemente confundidas (47).

- El job-sharing hace referencia a dos trabajadores a tiempo parcial, con una cualificación similar, que se dividen las horas, responsabilidades y beneficios de un trabajo a tiempo completo. En esta fórmula, cada uno de los componentes de la bina, se responsabiliza sólo de sus propias tareas.

- El jab-pairing consiste en el reparto de un puesto de trabajo entre dos trabajadores igualmente cualificados. En este arreglo, cada uno de los miembros de la pareja es responsable de la totalidad de las tareas del puesto.

- El job-splitting connota dos trabajadores con cualificaciones diferentes, que se dividen un trabajo a tiempo completo en áreas o tareas, de acuerdo con la formulación y habilidad de cada uno de ellos.

Los sistemas de TC se encuentran ampliamente implantados en los Estados Unidos, en donde, según una encuesta llevada a cabo por la Conference Board sobre política de Gestión de Personal, aplicada a una muestra de 6.000 empresas norteamericanas, es una fórmula común en el $12 \%$ de los bancos, $11 \%$ de las compañías de seguros, $2 \%$ de las empresas de producción para su personal de producción ( $4 \%$ para el personal administrativo), etc.

En Gran Bretaña, el sistema de TC ha contado con el apoyo oficial. El Department of Employ británico incentiva a las empresas que apliquen fórmulas de TC, con una gratificación de 750 libras esterlinas, pagaderas en cuatro plazos, por cada puesto de trabajo creado, dentro de determinadas condiciones (48).

(47) Vid, FREASE, M. y ZAWACKI, R. A." "Job-sharing; an answer to productivity problems". The Personnel Administrator. October 1979, pp. 35-56.

BICKERSTAFFE, G., "Un puesto a medias es mejor que el desempleo para uno solo", International Management. Marzo 1982, pp. 22 y 23.

(48) BILSLAND, t. y EVANS, A., "How to introduce Job-splitting". IPM Publishing. London. 1982. 
Entre nosotros, el contrato de relevo, definido por el artículo 7 del R.D. 1991/1984 de 31 de octubre como aquel "que se concierta con un trabajador inscrito como desempleado en la correspondiente oficina de Empleo, para sustituir al trabajador de la Empesa que accede a la jubilación parcial (...) simultáneamente con el contrato a tiempo parcial que se pacta con este ultimo, limitado al tiempo que falte al trabajador sustituido para alcanzar la edad de jubilación ordinaria", puede ser considerado como una modalidad de TC. En este tipo de contrato, la incentivación económica se limita a una reducción de la cuota patronal a la Seguridad Social, del orden del $50 \%$ de las contingencias comunes, por un periodo de tiempo igual al de vigencia del contrato de relevo, condicionado a que, terminado el contrato de relevo, se opte por su conversión en trabajo a jornada completa y por tiempo indefinido.

Los sistemas de TC admiten estructuraciones muy diversas. Los tiempos de trabajo suelen ser iguales para uno y otro trabajador, o pueden no serlo. Es posible compartir un puesto entre dos trabajadores, o entre tres, o incluso más personas. También se pueden compartir dos puestos de trabajo entre tres trabajadores. Pero lo habitual es repartir las tareas de un puesto entre dos trabajadores, con tiempos de trabajo iguales.

Aún así, este arreglo admite varias modalidades. Por ejemplo:

- A trabaja por las mañanas y $\mathrm{B}$ lo hace por las tardes.

- A trabaja lunes, martes y miércoles por la mañana, mientras B lo hace el miércoles por la tarde, todo el jueves y el viernes.

- A trabaja lunes, miércoles y viernes, y $B$ lo hace el martes y jueves. A la semana siguiente se invierte el proceso.

- Etcétera.

En cualquier caso, la comunicación entre los que comparten un puesto de trabajo es fundamental, por lo que debe preverse un período de presencia simultánea de ambos trabajadores en el momento del relevo, para que puedan comunicarse las novedades, trasmitir las órdenes, asegurarse de la continuación de las tareas iniciadas y aún no concluidas, etc.

En Estados Unidos, los dos trabajadores que constituyen una pareja de TC, firman un solo contrato de trabajo, mientras que en la RFA firman dos. Pero en uno y otro lugar esta previsto que:

- Si uno de los dos abandona el puesto, el otro debe abandonarlo también.

- La pareja es solidariamente responsable del puesto de trabajo. Si uno se retrasa en el momento del relevo, el otro no puede marcharse en tanto no compareza su compañero. Si uno de los dos está enfermo, o de permiso, el otro debe suplir su ausencia (49).

(49) Como era de esparar, esta cláusula suscitó una fuerte oposición por parte de los sindicatos, que acusaron a los empresarios de aprovecharse de las condiciones adversas coyunturales para reducir las conquistas sociales de los trabajadores. 
Las experiencias llevadas a cabo por parte de diferentes empresas en distintos países, permiten establecer un listado provisional de ventajas e inconvenientes de los sistemas de TC:

\section{Ventajas de los sistemas de TC}

- Incremento de la productividad, debido a que los trabajadores que sólo trabajan cuatro horas se fatigan menos que los que trabajan ocho, por tanto producen a un ritmo mayor.

- Reducción de errores y accidentes debidos a la fatiga.

- Reducción del absentismo, tanto injustificado como justificado.

- Reducción de gastos por horas extraordinarias.

- Reducción de gastos por formación. Cuando se forma a una pareja que va a compartir un puesto, el coste es ligeramente superior a formar uno sólo, pero cuando uno de los dos se marcha, el que permanece se encarga de la formación on-the-job de su nuevo compañero. $Y$ lo hace con gran interés, según parece, ya que su permanencia en el puesto depende de encontrar un nuevo compañero idóneo.

- Reducción de las dimisiones. Como los empleados que comparten un puesto han conseguido un horario a la medida de sus necesidades, es menos probable que se marchen.

- Buena calidad del trabajo realizado, como consecuencia del deseo de mostrar apreciación por su única oportunidad de encontrar empleo.

- Posibilidad de que puedan trabajar mujeres casadas, personas de más de cincuenta años y disminuidos físicos, que pueden desarrollar un esfuerzo por unas pocas horas, pero no por una jornada completa.

- Posibilidad de disminuir el número de parados, sin incrementar el número de puestos de trabajo.

\section{Inconvenientes de los sistemas de TC}

- Necesidad de "casar" los temperamentos de los que comparten un puesto de trabajo, para evitar futuros problemas.

- Dificultad de promoción para los que realizan un TC.

- Dificultad de compartir puestos de alto nivel.

- Rechazo de los directivos hacia este sistema.

\subsubsection{SOLUCIONES BASADAS EN LA RECONVERSION INTERNA} den ser:

Comprende este tipo de soluciones medidas tan dispares como pue-

- La movilidad geográfica.

- La movilidad funcional.

- La reconversión profesional:

- reconversión de unos oficios en otros; 
- reconversión de Mandos Intermedios en obreros;

- reconversión de administrativos en obreros.

- El trabajo domiciliario.

a) La movilidad geográfica. Se fundamenta esta práctica en la posibilidad o potestad de la empresa, previa la correspondiente convalidación administrativa, de trasladar a un trabajador de un puesto de trabajo situado en una localidad determinada, a otro, perteneciente a la misma empresa, pero situado en localidad distinta (50).

A pesar de las posibilidades que la legislación vigente otorga al empresario para la organización y la dirección de la actividad, es indudable que la aplicación de esta medida con carácter forzoso origina graves problemas a los trabajadores afectados. Problemas de gastos de traslado y de instalación, pero sobre todo, problemas humanos, tales como pérdida de las relaciones con el entorno, dificultades de "encaje" en la nueva localidad, problemas con los hijos, que quizá no puedan proseguir en el nuevo destino unos estudios iniciados en el anterior, etc. Sin embargo, en los casos en que se va a proceder al cierre de una sucursal, una delegam ción o una planta industrial, es posible que una parte del personal opte por un puesto de trabajo en otro lugar, en vez de percibir una indemnización por despido.

b) La movilidad funciona/ denota la posibilidad de que un trabajadoro empleado pueda ser destinado a una función distinta de la que venía realizando, por necesidades del servicio (51). A veces esta movilidad viene motivada por la desaparición de un taller o sección, o por disminución de la demanda. No es infrecuente que la introducción de nueva tecnología haga innecesario a parte del personal. En estos casos se suele optar por prejubilar o jubilar anticipadamente a los que se encuentran en edad para ello, y en reconvertír internamente a los más jóvenes.

OUCHI (52) afirma que, en Occidente, un hombre trabaja en diversas organizaciones, pero en una misma especialidad, mientras que en el Japón lo hace en diversas especialidades, pero dentro de una misma empresa. Si se le pregunta al hijo de un asalariado occidental qué hace su padre, la respuesta será: "Mi padre es ingeniero, o tornero, o administrativo." Si se hace la misma pregunta a un niño japonés, la respuesta previsible será: "Mi padre trabaja en la Compañía X."

La probabilidad de permanencia en una misma empresa y en un mismo puesto de trabajo, son posiciones inversamente proporcionales: cuando aumenta la una, disminuye la otra. En momentos de crisis o de reducción de plantilla, las mayores posibilidades de permanencia las tiene el empleado que posee en alto grado una cualidad, antes ignorada, pero que cada vez reviste mayor importancia en los procesos de selección: la polivalencia.

(50) E.T. art. 40.

(51) E.T, art. 41 .

(52) OUCHI. W. Op. cit. 
c) La reconversión profesional. Dentro de la reconversión interna, la medida de reconvertir obreros de una especialidad en otra, es la que menos problemas plantea. La reconversión de administrativos $y$, en general, de trabajadores "de cuello blanco" en obreros "de mono", es mucho más problemática. Y esto no tanto por el aspecto económico del cambio (muchos obreros "de mono" ganan más que los oficinistas) como por el choque psicológico que el cambio comporta.

Dentro de esta línea, la reconversión de mandos intermedios en obreros es mucho más complicada, precisamente por las implicaciones psicológicas de la reconversión. Aunque se les mantenga la remuneración original, el descenso de categoría, comparable a una degradación en el ejército, lleva a muchos M.I. reconvertibles a preferir un despido (53).

d) E/ trabajo domiciliario. Digamos de entrada que la posibilidad de "descontratar" trabajadores de plantilla y pasarlos a trabajar a domicilio, eximiéndose de obligaciones fiscales y de cotización a la Seguridad Social, es - naturalmente- ilegal.

Por otra parte, la contratación de nuevos trabajadores a domicilio, parece no aportar a la empresa ventajas económicas, puesto que, tanto desde el punto de vista salarial, como desde la perspectiva de las cotizaciones a la Seguridad Social, los costes son como mínimo, idénti$\cos (54)$.

Sin embargo, tanto una como otra posibilidad se dan a diario en muchos paises, y entre ellos en el nuestro. Esta situación está especialmente extendida en las industrias del calzado, confección, juguete, cuero y joyería, y localizada principalmente en Cataluña y la Comunidad Valeciana.

La crisis económica, el aumento de la presión fiscal, el paro, son señalados como causantes principales de este paso de industrias, otrora legales a la economía sumergida (55).

Datos recientes del Ministerio de Economía y Hacienda (Dirección General de Previsión y Coyuntura) afirman que, de los 650.000 trabajadores a domicilio existentes, sólo 142.000 (el $22 \%$ aproximadamente) cotizan a la Seguridad Social.

(53) Referencias personales de directores de personal que han aplicado esta medida. señalan que la resistencia disminuye cuando:

- La reconversion se conjuga con un traslado a otro centro de trabajo donde el M. I. no sea conocido.

- El nuevo trabajo es de tipo relativamente independiente.

- No se requiere llevar "mono" azul (una bata blanca es mucho mejor tolerada).

En algunos casos, los reconvertidos exigian seguir votando en las elección sindicales por el colegio de técnicos y administrativos en lugar de por el de trabajadores especialistas y no cualificados (!!!).

(54) E.T. art. 13.3 y Ley General de ta S. S. art. 7.1. a.

(55) Vid. NICOLAU. R., “La economia subterránea”. Alta Dirección, n. 104 1982, pp. 198 y siguientes.

MISSE, A. "El Tercer Mundo en casa". El Pais. 28.10.1984, pp. 66 y 67. 
Efectivamente, esta situación plantea problemas legales y económicos, de los que no es el menor la competencia desleal que hacen a las empresas que, por cotizar a la S.S., y pagar sus impuestos, tienen una mayor incidencia de los costes directos de personal sobre el precio final del producto, to que les hace salir al mercado en condiciones de inferioridad respecto de las empresas ilegales.

A veces se intenta justificar, no obstante, la economía subterránea o sumergida, argumentando que se trata de industrias que trabajan fundamentalmente para la exportación, y que tiene que competir con productos "made in Taiwan" o "made in Korea", países éstos en donde los costes salariales son apenas del orden del $20 \%$ respecto de los españoles, y que de no hacerlo así, la competencia sería imposible, las empresas cerrarían, el número de parados "reales" aumentaría, y el país se vería privado del aporte de divisas que la exportación de tales productos representa, con lo que el problema social, económico y humano sería mucho más grave.

Pero es que, además, este movimiento de economía subterránea, propio de países en crisis, coincide con otro movimiento paralelo, éste propio de países desarrollados, de incremento del trabajo domiciliario.

En la sociedad precapitalista, domicilio del patrono y lugar de trabajo era todo uno. Con el desarrollo de la industrialización, se separa el domicilio del patrono y la factoría, pero los procesos productivos exigen que todos los obreros, simultáneamente, coincidan en el taller. Actualmente, las nuevas tecnologías, que han reducido de manera drástica el número de trabajadores que tienen que manipular la mercancía física, unido al desarrollo de las comunicaciones, la electrónica y la informática, permiten sin demasiados problemas, el desplazamiento del lugar de trabajo al propio domicilio del trabajador, en porcentajes cada vez más elevados de asalariados (56).

No se puede, en absoluto, admitir como ajustada a una conducta ilícita, pero es forzoso reconocer que el problema del trabajo domiciliario es mucho más complejo de lo que puede parecer a primera vista, y que la desaparición radical de estas soluciones ilegales, podría causar más mal que bien.

En todo caso, cabe replantearse si el trabajo domiciliario "legal", es decir, aquel en que respetan todas las obligaciones fiscales, retributivas y de cotización a la Seguridad Social, es susceptible de reportar algún ahorro a la empresa, porque - recordémoslo una vez más - los procesos sustractivos no son un fin en sí mismos, sino un simple medio para aligerar los costes salariales directos e indirectos, y su consiguiente repersución sobre el precio final del producto o servicio.

El trabajo a domicilio "legal" no disminuye los costes actuales del trabajo, incluso encarece algo los indirectos (las reparaciones hay que

(56) Vid. TOFFLER, A., "La tercera ola". Plaza y Janés. Barcelona. 1980. pp. 198 y siguientes.

HERTEAUX, M. "El futuro del trabajo está en casa". El Pais. 16.9.1984. 
hacerlas a domicilio, así como el adiestramiento y la supervisión, el reparto de materia prima y la recogida del trabajo terminado). Sin embargo, se reduce la necesidad de incrementos salariales futuros, en razón de la reducción de gastos que el trabajador experimenta como consecuencia de trabajar en su propia casa.

Los costes a que nos referimos, son soportados directamente por el trabajador, pero naturalmente, son repercutidos al empresario en forma de salarios, y por último al consumidor, en forma de precios más elevados.

Así, el trabajador ahorra en:

- Transporte, capítulo éste que puede llegar a ser muy elevado, tanto si se utilizan los servicios públicos, como si se emplea vehículo propio.

- Comida. Comer fuera de casa siempre es más caro que comer en el propio domicilio.

- Ropa. El trabajador que realiza su tarea en casa, no precisa renovar su vestuario con tanta frecuencia.

- Tiempo, puesto que se invierte parte de él en traslados $y$ "horas muertas" (57).

Pero es que, además, hay un ahorro real directo por parte de la empresa, en capítulo tales como:

- Reducción de la conflictividad laboral, como resultado de la dispersión de los trabajadores y consecuentemente, menos horas perdidas por huelgas, paros, asambleas...

- Al realizarse el trabajo en casa, disminuye la necesidad de invertir en talleres, naves y oficinas para albergar a estos trabajadores, con la consiguiente reducción de inversiones en adquisición de solares, construcción de edificios, gastos de acondicionamiento y mantenimiento, decoración, calefacción y réfrigeración, limpieza, vigilancia... En el supuesto de que las instalaciones no sean propiedad de la empresa, reducción de los costes en concepto de alquiler de locales.

- Costes de aparcamiento para los coches de los trabajadores, de necesidad de guarderías para los hijos pequeños de los trabajadores, de mantenimiento de comedores de empresa...

El que habitualmente no se impute el coste de estas partidas a los costes salariales indirectos, no quiere decir que sean menos reales.

\subsection{ESTRATEGIAS Y TACTICAS PARA LA IMPLEMENTACION DE TODO TIPO DE SOLUCIONES SUSTRACTIVAS}

Dando por sentado que existen una serie de prejuicios adversos a cualquier medida tendente a la implantación de procesos sustractivos,

(57) La extensión de estos tiempos aumentan en sentido absoluto, por problemas de tráfico y de aparcamiento, pero más aún en sentido relativo. Esto es, a menos jornada laboral, mayor tiempo (proporcional) dedicado a desplazamientos. 
'tanto por parte de la dirección de la empresa y de los jefes más tradicionales, como por parte de los trabajadores, se hace imperativo planificar cuidadosamente la estrategia general y las tácticas particulares que han de ser aplicadas para reducir al mínimo los rechazos y conseguir la implicación de todas las partes afectadas. Las experiencias desarrolladas por diversas empresas, permiten recomendar, en principio, la utilización del esquema siguiente:

a) Realizar un estudio previo, en el que deben participar, necesaria. mente, los jefes de línea, en orden a identificar los puestos de trabajo que, en principio, pueden ser objeto de "descontratación", acondicionamiento del tiempo de trabajo o reconversión interna.

Esta fase tiene por objeto:

- Implicar a los jefes de línea.

- Detectar los puestos susceptibles de modificación.

- Descubrir los verdaderos impedimentos, en particular los de organización del trabajo, y eliminar los falsos obstáculos.

b) Presentar el provecto, una vez elaborado, al Comité de Empresa, para que lo estudien, y fijar un plazo para iniciar trabajos conjuntos en una comisión Comité de Empresa-Dirección de Personal.

Esta fase tiene por objeto:

- Implicar a los representantes de los trabajadores.

- Expresarles claramente los objetivos de la empresa.

- Identificar claramente las ventajas e inconvenientes que las fórmulas esbozadas revisten, tanto para el personal, como para la empresa.

Sería deseable que se trasladara a esta Comisión la idea de que no se trata de negociar nada, sino simplemente de trabajar conjuntamente, sin descartar - por supuesto - las necesarias negociaciones una vez el plan esté suficientemente elaborado.

Al final de esta etapa, debe haberse elaborado conjuntamente:

- Un cuestionario anónimo dirigido al conjunto del personal que permita conocer sus auténticas aspiraciones y problemas.

- Un esbozo de convenio de empresa, sobre las medidas concretas para la aplicación de los distintos programas. En el supuesto, nada improbable, de que las relaciones entre la dirección de la Empresa y los representantes de los trabajadores sean, o hayan sido muy tensas en el pasado, es preferible presentar el proyecto no al Comité de Empresa, sino a las propias Centrales Sindicales, y precisamente a sus asesores (jurídicos y económicos). La razón de proceder así es obvia: el técnico, tanto por su superior preparación como por su "distanciamiento" del problema, se encuentra en mejores condiciones que un miembro del Comité de Empresa para proceder con equidad y desapasionamiento. Se trata, pues, de evitar negativas viscerales a propuestas discutibles, pero viables. 
c) Aplicar a todo el personal el citado cuestionario anónimo, para conocer sus reacciones y su opinión acerca de las distintas fórmulas que se les podría ofrecer.

Esta fase pretende:

- Implicar a todo el personal.

- Tener una estimación global de los posibles interesados en cada una de las fórmulas ofrecidas.

- Recoger sugerencias aportadas por los interesados.

- Estimar el coste que la aplicaciór de cada una de las medidas supondría.

d) Consultar a la Inspección de Trabajo acerca de su postura sobre formulas que, sin ser ilegales, pueden resultar atipicas.

e) Elaboración de unas normas provisionales, difusión y "venta" de la idea a los peticionarios potenciales.

f) Aplicación piloto. Antes de embarcarse en una aventura cuyos resultados, de momento, son inciertos, es conveniente limitar la aplicación del plan a una unidad o taller. Los resultados obtenidos servirán para identificar y obviar los problemas de aplicación no previstos, incluso para modificar las normas provisionales.

Además, la restricción de la aplicación de la medida tiene un efecto psicológico: estimular más aún los deseos de participación del resto. Como dijo Víctor Hugo, "En amor, sigue y te huirán, huye y te seguirán".

g) Evaluación de los resultados obtenidos, rediseño o modificación de las medidas ofrecidas inicialmente, aplicación y generalización al resto de la empresa (58).

Como resumen, el éxito se alcanza cuando: sonal.

- Se acierta a detectar las aspiraciones y necesidades del per-

- Existe una implicación de todos (jefes, trabajadores, Comité de Empresa...).

- Hay una información previa, clara, concreta y completa, de modo que los objetivos y las reglas del juego estén claras para todos.

- Existe un abanico de fórmulas suficientemente amplio para que los posibles interesados encuentren satisfacción a sus problemas.

- Se consigue suscitar un clima favorable a la aplicación de las medidas.

- La Inspección de Trabajo conoce y no se opone a estas medidas, sin necesidad de que su no oposición se refleje necesariamente en un escrito, aunque esto átimo sería lo deseable.

(58) Este esquema se fundamenta, en parte, en el informe "Faciliter le developpement du travail à temps partiel dans une entreprise du secteur tertiaire". Personnel. Janvier $1983 . \mathrm{pp}$. 34-37, y en las informaciones de diversos responsables de personal de empresas españolas que han aplicado alguna medida de este tipo. 